\title{
Experimental Study of Light Propagation in Silicon Microring CROWs (Project Report 0925399-Y2)
}

\author{
SHAYAN MOOKHERJEA \\ Department of Electrical and Computer Engineering, University of California, San Diego, MC 0407 La \\ Jolla CA 92093-0407 USA \\ Email:smookher@ucsd.edu
}

The goal of this NSF-funded project [0925399 - Year 2] is to design, fabricate and study a new class of photonic circuits and devices in which disorder can be harnessed and controlled. There are two aspects to this project. Firstly, we will develop scientific insights in designing optical structures which can withstand or even utilize disorder for functionality. Secondly, we will make engineering advances in correcting or compensating for defective order.

\section{Introduction}

The principal focus of research during the second year (2010-11) of this project was on studying optical propagation in coupled-resonator devices which can comprise several hundred individual elements in a periodic structure along the direction of light propagation. We used this device structure as the foundation of this project because while there are simple models of propagation in the tightbinding regime [1]-[4], optical propagation near the regions of high dispersion [5] and high nonlinearity has not been easy to study [6]. Measurements made in this project were compared to our earlier theoretical models [7]-[9]. The coupled-resonator optical waveguide (CROW) structure can have many potential applications including low-power nonlinear optics [10]-[12], novel lasers [13] and generation of entangled photon pairs through spontaneous fourwave mixing (SFWM) [14]. Some of these advantages over traditional nonlinear optics using crystals can also be achieved using single microring resonators [15], and therefore a comparison of CROWs with single resonators is important.

\section{Activities}

Figure 1 shows an image of a device fabricated under this project and measured in our laboratory. CROWs of up to 235 coupled resonators were successfully fabricated and measured, and a good agreement was achieved between experiments and theory [16]-[18]. Infrared imaging is also a useful experimental tool to study light propagation through the length of these structures [19], [20]. A single point of failure in a directly-coupled CROW is sufficient to prevent any light propagation through the structure. However, we did not see any such catastrophic failures. Instead, the minor disorder between 
adjacent resonators causes ripples in the transmission spectrum which can be used for study and understanding the physics of light propagation in CROWs.

These CROW structures are based on microring resonators with directional couplers. We experimentally studied the dispersion of such couplers using a test structure fabricated at the university which consists of a microring coupled to a waveguide using a directional coupler [21]. An accurate knowledge of dispersion is necessary not only for CROW waveguides but also to design more compact devices such as mixers and filters in which the free-spectral ranges (FSR) of different sections must be precisely related [22]. We developed an extension of coupled-mode theory which is applicable to directional couplers in silicon photonics, coupled-waveguide structures [23] as well as coupledresonator structures [24]. Based on our analytical coupled-resonator models, we also developed a model for photon-pair generation using spontaneous fourwave mixing (SFWM) [25].

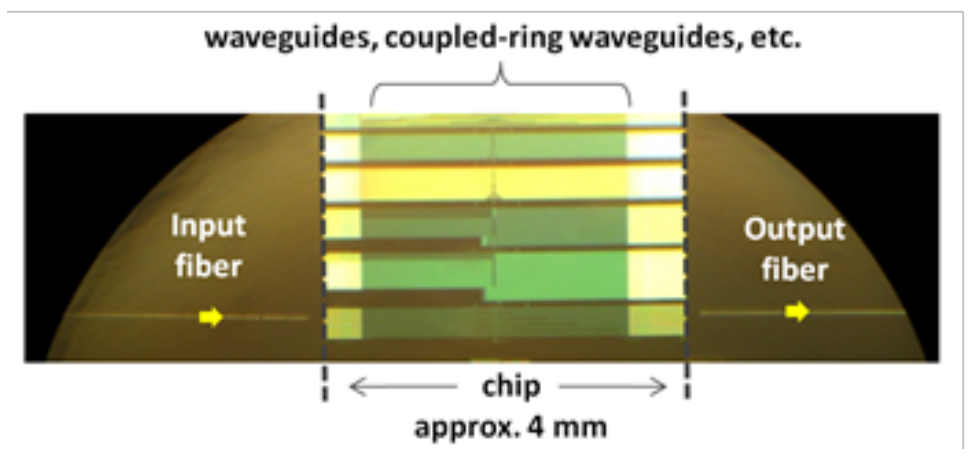

Figure 1 Microscope image of the silicon photonic chip and tapered optical fibers used for measurement. Four single-mode silicon waveguides of length $0.35 \mathrm{~cm}, 1.10 \mathrm{~cm}, 2.63 \mathrm{~cm}$, and $4.15 \mathrm{~cm}$, each having an equal number of bends, and five coupled ring waveguides/filters consisting of $35,65,95,135$ and 235 microring resonators were fabricated on each chip.

CROW structures shown in Figure 1 are passive waveguides. To achieve electronic control over optical propagation, we can use the electro-optic effect of silicon. CROW structures including the electro-optic effect [26] were designed and fabricated in a later project [27]. Alternatively, the nonlinear microring add-drop filter [28] could be considered as a building block for a tunable CROW device. We did not use this approach as it requires higher optical powers and may also be complicated by thermal effects. If tuning speed is not a concern, the thermo-optic effect obtained by simply heating the structure, which we used to demonstrate tunable filters based on CROWs, is also another control option [29]. Finally, optical gain could also be used to tune the dispersion relationship of CROWs but requires a material that can provide gain at these wavelengths [30].

\section{Progress and Impact}


Research accomplishments this year (2010-11) build on last year's milestone of fabricating long CROWs in silicon of up to 235 coupled microrings. These microring CROWs shown in Figure 1 were fabricated at the IBM Microelectronics Research Laboratory on $200 \mathrm{~mm}$ silicon-on-insulator (SOI) wafers with two different roughness reduction strategies. Coupled resonator optical waveguides consisting of 35, 65, 95, 135 and 235 microrings were fabricated with a nominal ring-to-ring coupling coefficient of $\kappa^{2}=0.15$. The bending radius of the rings was $6.5 \mu \mathrm{m}$, and it was verified that only a single polarization of light (TE) was transmitted. We measured wavelength-resolved optical transmission and group delay using an optical vector analyzer instrument (Luna Technologies) with $1.4 \mathrm{pm}$ resolution over $80 \mathrm{~nm}$ total bandwidth. High-speed modulated light (NRZ data modulation at $20 \mathrm{Gbps}$ ) was successfully transmitted through several passbands of a 235-ring CROW device (see Fig. 2). In prior work, data modulation at only a few Gbps could be transmitted through CROWs without significant distortion [31]. In contrast to previous studies, this shows that the average group delay ripple (GDR) was greatly improved in CROW structures that are hundreds of unit cells in length.

Of interest here is whether or not transmitted photons display unconventional statistical behavior. In particular, it is important to realize that measurement of only the average properties of the waveguide constitutes an incomplete story of transport. Instead, a distinction between ballistic, diffusive and localized transport regimes can be obtained only through an analysis of the probability distribution functions (PDFs) of the transmission and propagation time. This is a technique well known e.g., in turbulent atmospheric propagation, or light scattering through tissues or milky fluids. We believe we are among the first to apply such methods to study on-chip silicon photonic structures which will eventually be intended for a signal processing or communications application.

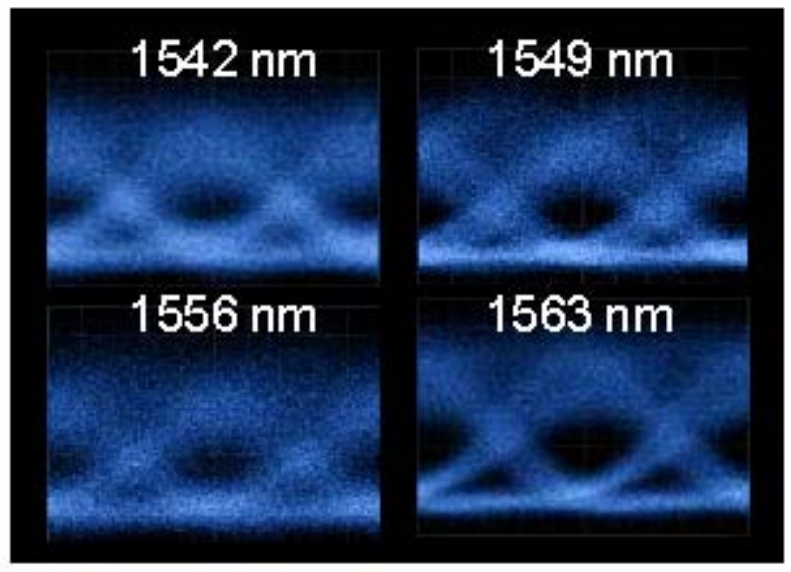

Figure 2 Eye diagrams of transmission of NRZ modulated light $(20 \mathrm{Gbps})$ at various passbands of a 235-ring CROW consisting of coupled silicon microring resonators. 
Figure 3(a) shows that in some CROWs, the statistical distribution of the normalized transmission intensity $(\hat{I} \equiv \mathrm{I} /<\mathrm{I}>$, the measured intensity normalized to its mean) was well described by a stretched-exponential decay functional which has the form

$$
P(\hat{I})=\exp [-2 \sqrt{g \hat{I}}] \text {, }
$$

where $\mathrm{g}=1.81 \pm 0.04$ was obtained by fitting the measured data. The behavior indicated by Eq. (1) is indicative of an intermediate physical transport regime which occurs between the well-known ballistic and localized regimes of light transport.
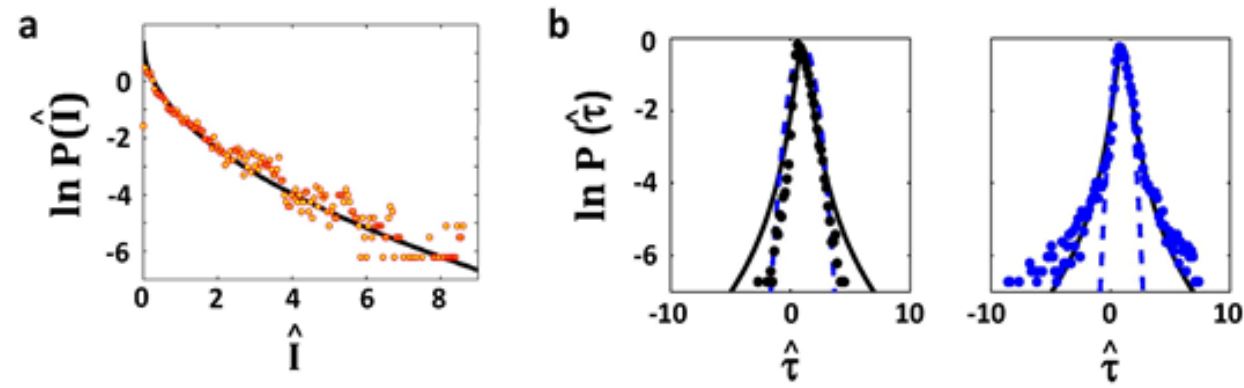

\begin{abstract}
Figure 3 (a) The distribution of normalized intensity, $\mathrm{I} /<\mathrm{I}>$, for a particular 235-ring coupledresonator waveguide was well fit by the stretched-exponential function, Eq. (1). (b) The probability distributions of $\tau /<\tau>$ are shown for the two waveguides of different length (35 rings and 65 rings) The dashed blue line is a Gaussian fit to the data, which indicates ballistic propagation statistics, and the solid black line is prediction of the distribution of single-channel time delay based on a diffusion model.
\end{abstract}

Figure 3(b) shows distributions of the normalized time delay of propagation $(\tau /<\tau>)$ for certain CROWs, which are plotted using a logarithmic scale for the vertical axis for clarity. Whereas the delay time distribution in the left-hand side panel of Fig. 2(b) was well described by a Gaussian fit, which is characteristic of the ballistic propagation regime, the distribution for the longer waveguide, shown in the right-hand side panel in Fig. 2(b) was more closely described by a parameter-free functional form used to describe a quasi-diffusive propagation regime [8], [32].

To improve the performance of coupled-resonator optical waveguides, we use an insight attributable to Van der Ziel nearly 60 years ago, that correlated electron flow results in less noise than the shot noise limit, to investigate mitigations of localization in long linear chains of optical resonators. The gray circles [panel (a)] and lines [panel (b)] in Fig. 4 correspond to simulations performed of optical transport for a typical random uncorrelated disorder distribution in a coupled resonator chain. The red squares and lines correspond 
to a disorder of the same magnitude but with non-zero spatial correlation. Fig. $3 \mathrm{~b}$ shows the difference in the simulated transmission spectra after propagating through one thousand coupled resonators in the chain. While the partiallycorrected passband was not perfectly flat and open, as would be ideal, there was very significant improvement in the case of partially-correlated disorder. Instead of localization-impaired nearly-zero transmission, intensity throughput is higher by $30 \mathrm{~dB}$, i.e., a factor of 1000 and, despite the presence of unavoidable transmission ripples, a usable frequency bandwidth is evident.

a

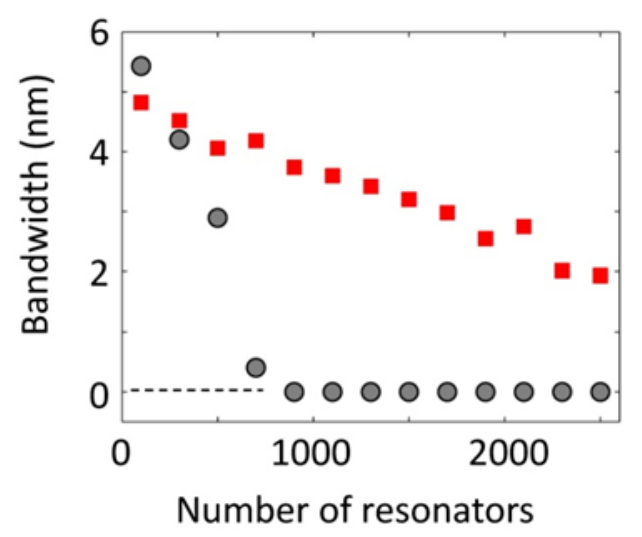

b

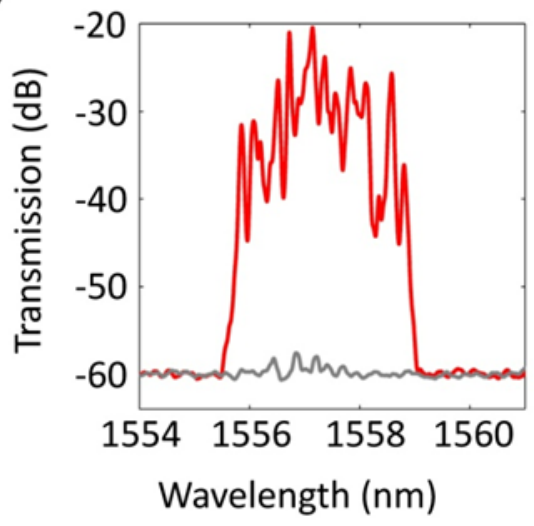

Figure 4(a), Simulations of bandwidth scaling in the case of uncorrelated disorder (gray circles) and partially-correlated disorder (red squares). The disordered potentials in the latter cases were modeled as the sum of $1 / 2$-times the uncorrelated-disorder potential and an additional correlateddisorder potential, whose variance was equal to that of the earlier (uncorrelated) distribution. (b) The simulated transmission after 1000 resonators was increased by nearly a factor of 1000 , showing the considerable benefits attainable by partial reduction of uncorrelated disorder, even if additional (correlated) disorder is added by an imperfect correction process.

External collaborations: A collaboration with IBM's silicon photonics group (Dr. W. M. J. Green) provided insights into silicon photonics fabrication and also supported technical collaboration on fabricating these structures. Several jointly-authored publications resulted from this collaboration [8], [32], [33]. Dr. Ivan B. Divliansky (Research Scientist, CREOL, Florida) collaborated with us for the electron-beam lithography of long waveguide structures. Jointlyauthored publications resulted from this collaboration [19]. A jointly-authored publication resulted from a collaboration [8] with Luna Technologies, VA.

Impact on Human Resources: Three students in the PI's group (one supported directly by this grant, one primarily supported by the PI's CAREER grant, and the third by an NSF Graduate Fellowship) have worked on this topic during the past year as part of their education and training. A post-doctoral scholar participated in this research and presented results at an invited talk at the SPIE Photonics West 2010 conference. Three undergraduates participated in summer 
research activities related to this project. One of the undergraduates was a Regents Scholar at UCSD and continued his research during the academic year.

\section{Subsequent Extensions}

The resonance frequency of a microring resonator is randomly shifted by hundreds of gigahertz because of few-nanometer variations in the width or height, commonly encountered during device fabrication, and these errors are enough to completely detune a filter or modulator from the intended operating

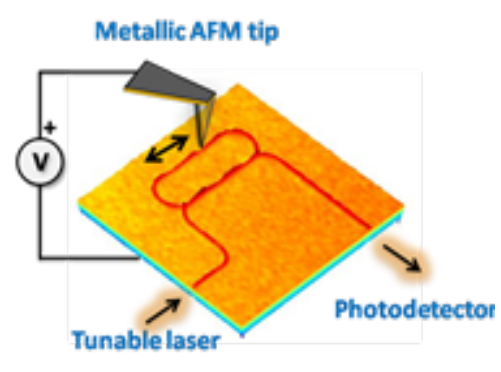

Figure 5 AFM nano-

oxidation trimming of a

silicon microring

resonator [24,25] frequency and also cause errors in MachZehnder modulator biasing and optical filters [34]. If nanoscale disorder can be modified, device performance may be improved. During this year, we have demonstrated this functionality as a natural extension of the research supported by this project.

Our method is based on field-induced local oxidation of $\mathrm{Si}$ to $\mathrm{SiO}_{2}$ via a chemical reaction near an electrically-biased conducting atomic-force microscope tip (see Fig. 5) [35], [36]. An electrically biased atomic force microscope (AFM) tip was scanned over the top surface of a silicon waveguide. This generated nanoscale oxide features by a process of electrochemical oxidation: conversion from $\mathrm{Si}$ to $\mathrm{SiO}_{2}$ is locally induced beneath the tip on the sample surface. The process is fairly insensitive to the tip shape and the ambient conditions. An adsorbed nanoscale water column below the tip is conjectured to play a role in the oxidation chemistry. For convenience, we used an AFM instrument available in our lab, but no part of our method depends on "atomic forces". In fact, similar scanning probe lithography has previously been used to create nanometer-scale oxide tunnel barriers in silicon and oxide electronic devices, modifying the resonance frequency of a GaAs photonic crystal cavity, and is part of a larger class of scanning probe lithographic methods developed for physical and chemical surface modification.

Each scan over a $9 \mu \mathrm{m}$ long waveguide section takes only a few seconds, and creates an index change $\left(\Delta \mathrm{n}_{\text {eff }}=-1.55 \times 10^{-4}\right.$ per scan) because of a permanent change of a thin layer (effective thickness $0.95 \AA /$ scan) of the waveguide core material (silicon) into cladding (silicon dioxide). The process appears to be stable, controllable, introduced no new materials. 
We studied and compared the single-resonator and coupled-resonator configurations from the viewpoint of quantum light generation [25]. The resonator-enhanced nonlinearity benefits the generation of entangled photonpair and heralded single-photon generation using silicon photonics [37]-[39]. Subsequently, it was seen that a single silicon microresonator can be monitored and controlled more easily [40] since it is difficult to manage many electronic wires and wirebonds in a long CROW device. An optimized single silicon microresonator can be a very useful device for nonlinear wavelength conversion with low pump powers [41]. The optical Kerr nonlinearity which is used as the physical principle for FWM and SFWM can also be used in other studies of nonlinear optics e.g., in the study of spatial solitons which are confined to a small region without requiring periodic lithographic patterning into a CROW or photonic crystal structure [42], [43].

\section{Open-Access Reporting Initiative}

PRAISE: This open-access document is provided in support of our PRAISE (Public Report of Activities, Impact and Subsequent Extensions) initiative. What is it? An open-access document shared with the public which describes the research outcomes of publicly-funded projects. For us, these projects are typically funded by the NSF (National Science Foundation).

\section{References}

[1] S. Olivier et al., "Miniband transmission in a photonic crystal coupledresonator optical waveguide," Opt. Lett., vol. 26, no. 13, pp. 1019-1021, 2001.

[2] T. Mukaiyama, K. Takeda, H. Miyazaki, Y. Jimba, and M. KuwataGonokami, "Tight-binding photonic molecule modes of resonant bispheres," Phys. Rev. Lett., vol. 82, no. 23, pp. 4623-4626, 1999.

[3] S. Mookherjea and A. Yariv, "Optical pulse propagation in the tightbinding approximation," Opt. Express, vol. 9, no. 2, p. 91, Jul. 2001, doi: 10.1364/OE.9.000091.

[4] N. Stefanou and A. Modinos, "Impurity bands in photonic insulators," Phys. Rev. B, vol. 57, no. 19, pp. 12127-12133, May 1998, doi: 10.1103/PhysRevB.57.12127.

[5] W. J. Kim, W. Kuang, and J. D. O'Brien, "Dispersion characteristics of photonic crystal coupled resonator optical waveguides," Opt. Express, vol. 11, pp. 3431-3437, 2003.

[6] S. Mookherjea, D. S. Cohen, and A. Yariv, "Nonlinear dispersion in a coupled-resonator optical waveguide," Opt. Lett., vol. 27, no. 11, p. 933, Jun. 2002, doi: 10.1364/OL.27.000933. 
[7] S. Mookherjea and A. Yariv, "Pulse propagation in a coupled resonator optical waveguide to all orders of dispersion," Phys. Rev. E, vol. 65, no. 5, p. 056601, Apr. 2002, doi: 10.1103/PhysRevE.65.056601.

[8] M. L. Cooper et al., "Waveguide dispersion effects in silicon-on-insulator coupled-resonator optical waveguides," Opt. Lett., vol. 35, no. 18, p. 3030, Sep. 2010, doi: 10.1364/OL.35.003030.

[9] S. Mookherjea, "Dispersion characteristics of coupled-resonator optical waveguides," Opt. Lett., vol. 30, no. 18, p. 2406, Sep. 2005, doi: 10.1364/OL.30.002406.

[10] S. Mookherjea and A. Yariv, "Second-harmonic generation with pulses in a coupled-resonator optical waveguide," Phys. Rev. E, vol. 65, no. 2, p. 026607, Jan. 2002, doi: 10.1103/PhysRevE.65.026607.

[11] S. Mookherjea and A. Yariv, "Kerr-stabilized super-resonant modes in coupled-resonator optical waveguides," Phys. Rev. E, vol. 66, no. 4, p. 046610, Oct. 2002, doi: 10.1103/PhysRevE.66.046610.

[12] S. Mookherjea and A. Yariv, "Optical pulse propagation and holographic storage in a coupled-resonator optical waveguide," Phys. Rev. $E$, vol. 64 , no. 6, p. 066602 , Nov. 2001, doi: 10.1103/PhysRevE.64.066602.

[13] S. Mookherjea, "Semiconductor coupled-resonator optical waveguide laser," Appl. Phys. Lett., vol. 84, no. 17, pp. 3265-3267, Apr. 2004, doi: 10.1063/1.1719278.

[14] R. Kumar, M. Savanier, J. R. Ong, and S. Mookherjea, "Entanglement measurement of a coupled silicon microring photon pair source," Opt. Express, vol. 23, no. 15, p. 19318, Jul. 2015, doi: 10.1364/OE.23.019318.

[15] C. Ma, X. Wang, V. Anant, A. D. Beyer, M. D. Shaw, and S. Mookherjea, "Silicon photonic entangled photon-pair and heralded single photon generation with CAR $>12,000$ and $\mathrm{g}^{\wedge}(2)(0)<0006$," Opt.

Express, vol. 25, no. 26, p. 32995, Dec. 2017, doi: 10.1364/OE.25.032995.

[16] M. L. Cooper et al., "235-ring Coupled-Resonator Optical Waveguides," in Conference on Lasers and Electro-Optics 2010, San Jose, California, 2010, p. CTuHH3. doi: 10.1364/CLEO.2010.CTuHH3.

[17] S. Mookherjea and M. A. Schneider, "Avoiding bandwidth collapse in long chains of coupled optical microresonators," Opt. Lett., vol. 36, no. 23, p. 4557, Dec. 2011, doi: 10.1364/OL.36.004557.

[18] M. L. Cooper and S. Mookherjea, "Modeling of Multiband Transmission in Long Silicon Coupled-Resonator Optical Waveguides," IEEE Photon. Technol. Lett., vol. 23, no. 13, pp. 872-874, Jul. 2011, doi: 10.1109/LPT.2011.2141657.

[19] M. L. Cooper, G. Gupta, J. S. Park, M. A. Schneider, I. B. Divliansky, and S. Mookherjea, "Quantitative infrared imaging of silicon-on-insulator 
microring resonators," Opt. Lett., vol. 35, no. 5, p. 784, Mar. 2010, doi: 10.1364/OL.35.000784.

[20] S. Mookherjea and H. R. Grant, "High dynamic range microscope infrared imaging of silicon nanophotonic devices," Opt. Lett., vol. 37, no. 22, p. 4705, Nov. 2012, doi: 10.1364/OL.37.004705.

[21] R. Aguinaldo, Yiran Shen, and S. Mookherjea, "Large Dispersion of Silicon Directional Couplers Obtained via Wideband Microring Parametric Characterization," IEEE Photon. Technol. Lett., vol. 24, no. 14, pp. 1242-1244, Jul. 2012, doi: 10.1109/LPT.2012.2198639.

[22] J. R. Ong, R. Kumar, and S. Mookherjea, "Silicon microring-based wavelength converter with integrated pump and signal suppression," Opt. Lett., vol. 39, no. 15, p. 4439, Aug. 2014, doi: 10.1364/OL.39.004439.

[23] M. L. Cooper and S. Mookherjea, "Numerically-assisted coupledmode theory for silicon waveguide couplers and arrayed waveguides," Opt. Express, vol. 17, no. 3, p. 1583, Feb. 2009, doi: 10.1364/OE.17.001583.

[24] S. Mookherjea, "Spectral characteristics of coupled resonators," $J$. Opt. Soc. Am. B, vol. 23, no. 6, p. 1137, Jun. 2006, doi:

10.1364/JOSAB.23.001137.

[25] J. R. Ong and S. Mookherjea, "Quantum light generation on a silicon chip using waveguides and resonators," Opt. Express, vol. 21, no. 4, p. 5171, Feb. 2013, doi: 10.1364/OE.21.005171.

[26] Q. Xu, B. Schmidt, S. Pradhan, and M. Lipson, "Micrometre-scale silicon electro-optic modulator," Nature, vol. 435, pp. 325-327, 2005.

[27] S. Mookherjea, J. R. Ong, X. Luo, and L. Guo-Qiang, "Electronic control of optical Anderson localization modes," Nature Nanotech, vol. 9, no. 5, pp. 365-371, May 2014, doi: 10.1038/nnano.2014.53.

[28] S. Mookherjea and M. A. Schneider, "The nonlinear microring adddrop filter," Opt. Express, vol. 16, no. 19, p. 15130, Sep. 2008, doi: 10.1364/OE.16.015130.

[29] R. Aguinaldo et al., "Wideband silicon-photonic thermo-optic switch in a wavelength-division multiplexed ring network," Opt. Express, vol. 22, no. 7, p. 8205, Apr. 2014, doi: 10.1364/OE.22.008205.

[30] S. Mookherjea, "Using gain to tune the dispersion relation of coupledresonator optical waveguides," IEEE Photon. Technol. Lett., vol. 18, no. 5, pp. 715-717, Mar. 2006, doi: 10.1109/LPT.2006.871144.

[31] F. Xia, L. Sekaric, and Y. Vlasov, "Ultracompact optical buffers on a silicon chip," Nature Photon, vol. 1, no. 1, pp. 65-71, Jan. 2007, doi: 10.1038/nphoton.2006.42.

[32] M. L. Cooper et al., "Statistics of light transport in 235-ring silicon coupled-resonator optical waveguides," Opt. Express, vol. 18, no. 25, p. 26505, Dec. 2010, doi: 10.1364/OE.18.026505. 
[33] J. R. Ong et al., "Low-power continuous-wave four-wave mixing in silicon coupled-resonator optical waveguides," Opt. Lett., vol. 36, no. 15, pp. 2964-2966, 2011.

[34] X. Wang, P. O. Weigel, J. Zhao, M. Ruesing, and S. Mookherjea, "Achieving beyond-100-GHz large-signal modulation bandwidth in hybrid silicon photonics Mach Zehnder modulators using thin film lithium niobate," APL Photonics, vol. 4, no. 9, p. 096101, Sep. 2019, doi: 10.1063/1.5115243.

[35] Y. Shen, I. B. Divliansky, D. N. Basov, and S. Mookherjea, "Electricfield-driven nano-oxidation trimming of silicon microrings and interferometers," Opt. Lett., vol. 36, no. 14, p. 2668, Jul. 2011, doi: 10.1364/OL.36.002668.

[36] Y. Shen, I. B. Divliansky, D. N. Basov, and S. Mookherjea, "Perfect set-and-forget alignment of silicon photonic resonators and interferometers," in Optical Fiber Communication Conference/National Fiber Optic Engineers Conference 2011, Los Angeles, California, 2011, p. PDPC3. doi: 10.1364/OFC.2011.PDPC3.

[37] M. Davanço et al., "Telecommunications-band heralded single photons from a silicon nanophotonic chip," Appl. Phys. Lett., vol. 100, no. 26, p. 261104, Jun. 2012, doi: 10.1063/1.4711253.

[38] R. Kumar, J. R. Ong, M. Savanier, and S. Mookherjea, "Controlling the spectrum of photons generated on a silicon nanophotonic chip," Nat Commun, vol. 5, no. 1, p. 5489, Dec. 2014, doi: 10.1038/ncomms6489.

[39] R. Kumar, J. R. Ong, J. Recchio, K. Srinivasan, and S. Mookherjea, "Spectrally multiplexed and tunable-wavelength photon pairs at $155 \mu \mathrm{m}$ from a silicon coupled-resonator optical waveguide," Opt. Lett., vol. 38, no. 16, p. 2969, Aug. 2013, doi: 10.1364/OL.38.002969.

[40] M. Savanier, R. Kumar, and S. Mookherjea, "Optimizing photon-pair generation electronically using a $p-i-n$ diode incorporated in a silicon microring resonator," Appl. Phys. Lett., vol. 107, no. 13, p. 131101, Sep. 2015, doi: 10.1063/1.4932047.

[41] J. R. Ong, R. Kumar, R. Aguinaldo, and S. Mookherjea, "Efficient CW Four-Wave Mixing in Silicon-on-Insulator Micro-Rings With Active Carrier Removal," IEEE Photon. Technol. Lett., vol. 25, no. 17, pp. 1699 1702, Sep. 2013, doi: 10.1109/LPT.2013.2272521.

[42] A. Ciattoni, B. Crosignani, S. Mookherjea, and A. Yariv, "Nonparaxial dark solitons in optical Kerr media," Opt. Lett., vol. 30, no. 5, p. 516, Mar. 2005, doi: 10.1364/OL.30.000516.

[43] B. Crosignani, A. Yariv, and S. Mookherjea, "Nonparaxial spatial solitons and propagation-invariant pattern solutions in optical Kerr media," Opt. Lett., vol. 29, no. 11, p. 1254, Jun. 2004, doi: 10.1364/OL.29.001254. 